\title{
Radar Target Discrimination Based on Co-null Polarization State:A Resonance Mode Context
}

\author{
Faisal Aldhubaib
}

\author{
Electronics Department, College of Technological Studies, Public Authority of Applied Education, Kuwait
}

\begin{abstract}
The concept of co-polarization nullstates is established ina resonance mode contextto describe a radar target of interest. This is achieved by Singularity Expansion Method to represent the target transient response byits natural resonance modes. Therefore the polarization description of the target is based on a quadrature residue matrixat each resonance mode.The copolarization null states are derivedby Lagranian optimization of the second moment properties of the quadrature residue matrix. $A$ wire model of aircraftisused to demonstrate the ability of conull states based resonanceto convey thetarget complex composite like elongation and tilt degrees.
\end{abstract}

Keywords: Characteristic polarization states, radar feature set, Kennaugh matrix, Stokes vector, Lagrange optimization, Singularity Expansion Method.

\section{Introduction}

In resonance based target recognition, studies have mainly been focus on exploiting the natural resonant modes embedded in an ultra-broadband transient signature measured at a specific aspect and polarization state[1-4], and sometime incorporated a polarization descriptor[5-8]. As the target works as a polarization transformer, information about the target polarization characteristics can be deduced. Robust but requires additional computation is the optimal or characteristic polarization states (CPS), whichrepresent the polarization statesof reception at which maximum, minimum, or null reception exist. For aco-polarized (co-pol) reception, there will be four CPS, namely two co-pol maximums $\left(\mathrm{cm}_{1,2}\right)$ and two co-pol nulls $\left(\mathrm{cn}_{1,2}\right)[9,10]$. Of which, the co-pol null states should be able to unfold the degrees of elongation and tilt in the target along the incidence direction.

However, the CPS concept has only been developed with the assumption that the backscattered wave has no or only small spectral dispersion, and therefore it is limited to completely polarized waves [11-13]. As a result the concept of using CPS to characterize a radar target is well developed in a narrowband context. This narrowband limitation can be tied to the fact that the target polarization response is a function of frequency and therefore spectrally non-uniform depolarization exists if impulsiveillumination andreception are used. Note, this is unlike the spectral spread due to noise, clutter, or even refraction in the transmission medium; all of which can be handled by ensemble or time averaging achieved by means of pulse integration $[14,15]$.

Toextend the CPS concept into a broadband context, a sparse representation (signature based-model) approach is required to find parameters from the target impulseresponse that are time-invariant, and at the same timecan relate robustly to the target composite. To achieve this, the singularitytheory, as defined in the Singularity Expansion Method (SEM)[16], is exploited. According to SEM, the late time portion of the temporal response from a perfect electric conducting (PEC) body can be represented in the time domain as aseries of resonance modes with natural frequencies and residues. The set of resonant frequency isrelated to the target dimension, have enhanced returns, and independent of aspect and polarization directions[17]. Whereas the set of resonance residue is dependent on aspect and polarization directions and thus they carry the polarization characteristic of the target.Therefore, the CPS can be derived using the complex residues, and subsequently, information about the target composite shape can be inferred from at least one co-null state in case ofa symmetrical target.

Previously, the author hasmade attempts to extend the CPS concept intobroadband context[18-22]. So the contribution this paper made is to use the co-pol null of the resonance modesto discriminate complex, but similar,radar targets.

\section{Model and Formulation}

\section{A. Second moment of Quadrature Residue}

If a radar target is illuminated by a sufficiently broadband excitation wave, with bandwidth covering the target dominant resonance modes, a suitable extraction algorithm such as the Method pencil-of-function (MPOF) [23], should be capable of recovering the targetresonance modes from the late time portion of the backscattered temporalresponse. According to the SEM model, the late time of the target impulse response can be expressed as a sum of damped complex exponentials, with dominant terms relating to the dimensions and composition of the target.

The received transient response in late time may be approximated according to SEM model as

$$
s(t)=\sum_{n=1}^{M} c_{n} e^{-\left(\sigma_{n}+j \omega_{n}\right) t}
$$

Here $t>T_{\mathrm{L}}$ where $T_{\mathrm{L}}$ denotes the late time onset after which the incident wave has totally passed the target and is approximated by twice the length of the target maximum dimension $L_{\max }$ per speed of wave, i.e. $T_{\mathrm{L}}=2 L_{\max } / \mathrm{c}$. The parameters $\sigma$, wandcrespectively denotea damping factor, a natural resonant frequency and a complex residue.The modal orderMgives the number of modes presumably excited. The parameters $\sigma$ and ware both insensitive to target aspect and polarization directions. On the contrary, the residuesare aspect and polarization dependent and hence reflect the target shape characteristics. Bearing in mind, that full polarization 


\section{International Journal of Science and Research (IJSR) \\ ISSN (Online): 2319-7064}

Index Copernicus Value (2013): 6.14 | Impact Factor (2015): 6.391

characteristics about a target, in general, are not contained at a single mode except for targets made of a single structure such as wires or spheres.

For quadrature polarization directions, the backscattered responses in orthogonal linear basis of transmission and reception directions form a real matrix $\mathbf{R}^{2 \times 2}$ as follows

$$
\mathrm{S}_{\mathrm{t}}=\left[\begin{array}{ll}
s_{x z}(t) & s_{x y}(t) \\
s_{y z}(t) & s_{y y}(t)
\end{array}\right]
$$

Where $x x$ and $y y$ denote the co-polarized scattering directions or channels, whilexy and $y x$ denote thecross-polarized scattering channels (reciprocal for monostatic case). Since a mode polarization characteristics is embedded in its quadrature resides, a residue matrix $C^{2 \times 2}$ that describes thetarget polarization scattering at a singleresonance can be derived by dropping the time dependence term $\exp \left(-s_{n} t\right)$ as follows

$$
\mathbf{C}=\left[\begin{array}{ll}
c_{x x} & c_{y x} \\
c_{x y} & c_{y y}
\end{array}\right]
$$

The subscripts denote the transmitter and receiver polarization directions, respectively, where the complex residues $\mathrm{c}_{x x}$ and $\mathrm{c}_{y y}$ denote the co-pol scattering coefficients; whereas $\mathrm{c}_{x y}$ and $\mathrm{c}_{y x}$ denote the $\mathrm{x}$-pol scattering coefficients and both are equal for reciprocal case (monostatic case). The complex exponential term $\exp (-s(n) . t)$ dependence was dropped because the scattering coefficients in a single matrix share acommon term.

More generally, a power measurement is used to avoid the coherent phase requirement of the residue matrix $\mathbf{C}$, and therefore, the Stokes vector is utilized to describe the polarization state of a wave or antenna for completely (and even partially) polarized waves as follows

$$
\mathbf{g}=\left[\begin{array}{llll}
g_{0} & g_{1} & g_{2} & g_{3}
\end{array}\right]^{\mathrm{T}}
$$

Subject to: $g_{0}=\left(g_{1}{ }^{2}+g_{2}{ }^{2}+g_{3}{ }^{2}\right)^{1 / 2}$ for fully polarized wave. ${ }^{\mathrm{T}}$ denotes the transpose. The $g_{0}$ denotes the wave intensity or total instantaneous power, $\mathrm{g}_{1}$ gives the portion of the wave that is horizontally or vertically polarized, $g_{2}$ gives the portion of the wave that is linearly oriented at $\pm 45^{\circ}$ and $g_{3}$ gives the portion of the wave that is left or right circularly polarized, respectively [16]. In the case of a partially polarized wave due to clutter or noise, ensemble-time averaging of Stokes vector is used.

In general, the second moment properties (i.e. power terms) of the residue matrix can be represented by the Kennaughformulism, as follows [17]

$$
[\mathrm{K}]=\mathbf{R}\left(\left|\mathbf{C} \otimes \mathrm{C}^{*}\right|\right) \mathbf{R}^{-1}, \quad \mathbf{R}^{-1}=\frac{1}{2} \mathbf{R}^{* T}
$$

Here

$$
\mathbf{R}=\left(\begin{array}{cccc}
1 & 0 & 0 & 1 \\
1 & 0 & 0 & -1 \\
0 & 1 & 1 & 0 \\
0 & j & -j & 0
\end{array}\right)
$$

The product $\left(\left|\mathbf{C} \otimes \mathbf{C}^{*}\right|\right)$ gives the entire correlation relations between the quadrature residues as follows

$$
\mathbf{C} \otimes \mathbf{C}^{*}=\left(\begin{array}{lllll}
c_{x x} * c_{x x}{ }^{*}, & c_{x x} * c_{x y}{ }^{*}, & c_{x y} * c_{x x}{ }^{*}, & c_{x y} * c_{x y}{ }^{*} \\
c_{x x} * c_{y x}{ }^{*}, & c_{x x} * c_{y y}{ }^{*}, & c_{x y} * c_{y x}{ }^{*}, & c_{x y} * c_{y y}{ }^{*} \\
c_{y x} * c_{x x}, & c_{y x} * c_{x y}{ }^{*}, & c_{y y} * c_{x x}, & c_{y y} * c_{x y}{ }^{*} \\
c_{y x} * c_{y x}, & c_{y x} * c_{y y}{ }^{*}, & c_{y y} * c_{y x}{ }^{*}, & c_{y y} * c_{y y}{ }^{*}
\end{array}\right)
$$

In general, the Kennaugh matrix relates incident and scattered Stokes vectors $\mathbf{g}_{\mathbf{i}}$ and $\mathbf{g}_{\mathbf{s}}$ as follows

$$
g_{s}=K \cdot g_{i}
$$

Power Optimization by Lagrange Multipliers

For backscattering and in the reciprocal case, it was pointed out in $[10,14]$ that the use of one antenna for transmitting and receiving is adequate to give maximum power and no greater power can be received in separate antennas for transmitting and receiving. Thus the one antenna case (monostatic case) assumed where the antenna Stokes vector $\mathrm{g}_{\mathrm{a}}$ is identical for transmit and receive and consequently the incident wave Stokes vector $\mathrm{g}_{\mathrm{i}}$ is identical to the antenna Stokes vectorga. Therefore the power at the receiver terminals can be expressed in terms of $g_{i}$ and $\mathrm{K}$ as

$$
P_{\mathrm{c}}=\mathrm{g}_{\mathrm{i}} \cdot \mathrm{g}_{\mathrm{s}} \mathrm{T}^{\mathrm{T}} \mathrm{g}_{\mathrm{i}} \cdot[\mathrm{K}] \cdot \mathrm{g}_{\mathrm{i}}^{\mathrm{T}}
$$

The associated received power in the co-pol channel is

$$
P_{c}=\mathbf{g}_{\mathbf{a}} \cdot\left[\mathbf{K}_{c}\right] \cdot \mathbf{g}_{\mathrm{a}}^{\mathrm{T}}
$$

Where

$$
\left[\mathbf{K}_{\mathrm{c}}\right]=\frac{1}{2}\left(\begin{array}{cccc}
1 & 0 & 0 & 0 \\
0 & 1 & 0 & 0 \\
0 & 0 & 1 & 0 \\
0 & 0 & 0 & -1
\end{array}\right) \cdot[\mathbf{K}]
$$

Here the subscript , ,ee denotes the co-pol configuration. Equation (8) is essential for deriving the co-pol CPS and their associated power levels using the Lagrangian multiplier method.

For the one antenna case (monostatic case), optimization involves properly choosing the antenna polarization states such that power developed at the receiving antenna terminals is maximum, minimum, or null. The power optimization will be carried out for the co-pol only, since all the target physical attributes can be inferred from the co-pol CPS.Without loss of generalization, the received power in (8)can be maximized and minimized by applying the Lagrangian multiplier method to the incident Stokes vector $\mathbf{g}_{\mathbf{i}}$ with the constraint that the transmit power is normalized to unity. The constraint condition $\Phi$ written in terms of the Stokes variables $\left(\mathrm{g}_{1}, \mathrm{~g}_{2}, \mathrm{~g}_{3}\right)$ is then defined as

$$
\Phi\left(g_{2}, g_{2}, g_{3}\right)=\sqrt{g_{1}^{2}+g_{2}^{2}+g_{3}^{2}}-1=0
$$




\section{International Journal of Science and Research (IJSR) \\ ISSN (Online): 2319-7064}

Index Copernicus Value (2013): 6.14 | Impact Factor (2015): 6.391

The variations of the incident Stokes variables will lead to maximizing or minimizing the received power at the antenna terminals, where the optimum co-pol powers can be found by simultaneously solving for the first partial derivatives of (8) subject to the constraint condition of (9).This procedure results in three simultaneous partial derivative equations as follows:

$$
\frac{\partial P_{c}}{\partial g_{m}}-\mu \frac{\partial \Phi}{\partial g_{m}}=0, \quad m=1,2,3
$$

Here $\mu$ is the Lagrangian multiplier which gives the rate of change of the power quantity being optimized as a function of the constraint variables. Solving (10)produces two pairs of co-pol CPS. These are the orthogonal co-pol max pair $\left(\mathrm{cm}_{1,2}\right)$ and the co-pol null pair $\left(\mathrm{cn}_{1,2}\right)$ surrounding the smallest $\mathrm{cm}$. These four states will form a polarization fork shape around a great circle as depicted in Figure 1. The latter are not orthogonal but define a characteristic angle, andthe dot product $\mathrm{g}_{\mathrm{cn} 1} \cdot \mathrm{g}_{\mathrm{cn} 2}$ is a measure of the elongation degree (1 for wire; -1 for sphere); whereas the symmetry information is reflected in $\mathrm{g}_{3}$ of the maximum $\mathrm{cm}$ ( 0 for symmetrical target, \pm 1 for totally nonsymmetrical target). The variablesg ${ }_{1}, g_{2}$ of the sum $\left[\mathrm{g}_{\mathrm{cn} 1}+\mathrm{g}_{\mathrm{cn} 2}\right]$ determine the tilt angle of a geometry as $1 / 2 \tan ^{-1}\left(g_{2} / g_{1}\right)$.

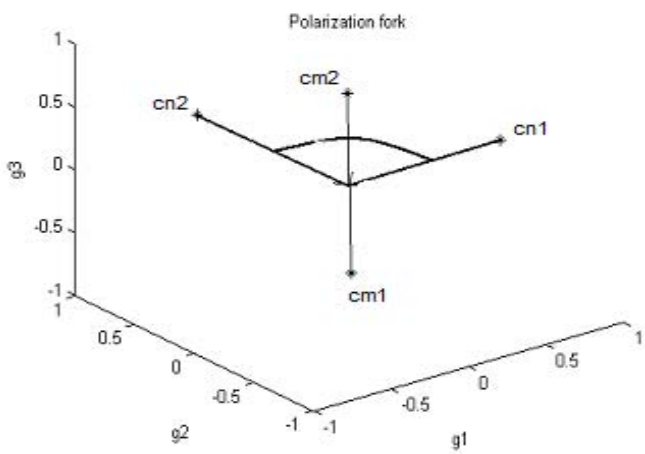

Figure 1: Characteristic polarization state pairs form a polarization signature (polarization fork)

\section{Numerical Example}

To validate the concept of describing the target substructures bythe CPS in a transient context by means of the SEM in a time approach, a numerical example using a metallic wire modelof aircraft is implemented. The modeled aircraft and the simulation values are depicted in Error! Reference source not found.. The angle $\theta_{w}$ defines the wings inclination angle while $\theta_{t}$ defines the tails inclination angle with the longitudinal axis. In this case, two models with two different inclination set $\left(\theta_{w}, \theta_{t}\right)=\left(45^{\circ}, 90^{\circ}\right)$ and $\left(90^{\circ}, 45^{\circ}\right)$ are used to validate the robustness of the co-nulls to discriminate between both model. Changing the angles $\theta_{w}$ and $\theta_{t}$ has no effect on the geometries dimensions (i.e. similar set of resonant frequencies) but leads to different shapes, and subsequently, different polarization characteristics. The transverse direction angle of the wave fields were set at $+45^{\circ}$ and $+135^{\circ}$ from the model longitudinal axis, i.e. the target is rotated at $45^{\circ}$ clockwise about the wave fields axes. This setup of polarization basis will insure that the target returnswithin any polarization channel is not null, and this will minimize the problem of omitted or misaligned residue in the quadrature residue matrix [22].

The simulated backscattered frequency-domain data were generated by method of moments algorithm (MoM) using FEKO[24]. The resultant backscattered was filtered by a Gaussian window to create the effect of a Gaussian shaped impulse and then the frequency data were transformed to the time-domain by inverse Fourier transform.Figure 3depicts a sample of the frequency responses for co-pol and x-pol channels, however it does not give much insight into the aircraft scattering mechanisms. InFigure 4 and Table I, the aircraft sections are labelled by their segment numbers, and their detailed current vs. frequency responses are plotted in Figure 5 and Figure 6. This provides a breakdown of the aircraft resonant frequencies (as indicated by the relevant peaks in the spectrum), and indicates to which geometrical section a resonance belongs. The estimated resonance set is at $150,300,410$ and $490 \mathrm{MHz}$ and respectively belong to mid, nose-wing, wing and tail.



Figure 2: Dimensions (in $\mathrm{cm}$ ) of the generalized aircraft model. The angles $\theta_{\mathrm{w}}$ and $\theta_{\mathrm{t}}$ give the model different shapes, but do not change the model dimensions.

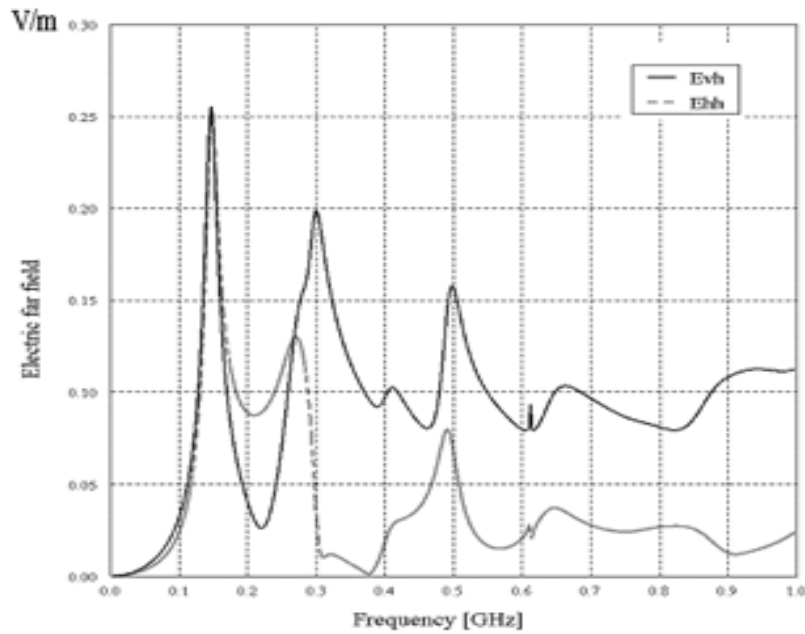

Figure 3: Aircraft frequency spectra in hhand $v h$ polarization channels. Spectrum peaks reflect the aircraft resonant frequencies to a great degree 


\section{International Journal of Science and Research (IJSR) \\ ISSN (Online): 2319-7064}

Index Copernicus Value (2013): 6.14 | Impact Factor (2015): 6.391



Figure 4: Aircraft model sections labeled in term of segment number

Table I: Aircraft major geometries labeled in terms of

\begin{tabular}{|c|c|c|}
\hline \multicolumn{3}{|c}{ segment number } \\
\hline Geometry & Start Segment \# & End Segment\# \\
\hline Nose & 1 & 11 \\
\hline Wings & $(15),(28)$ & $(26),(39)$ \\
\hline Mid & 42 & 56 \\
\hline Tail & $(69,78)$ & $(74,83)$ \\
\hline Tail Stabilizers & $(66),(75)$ & $(74),(83)$ \\
\hline
\end{tabular}

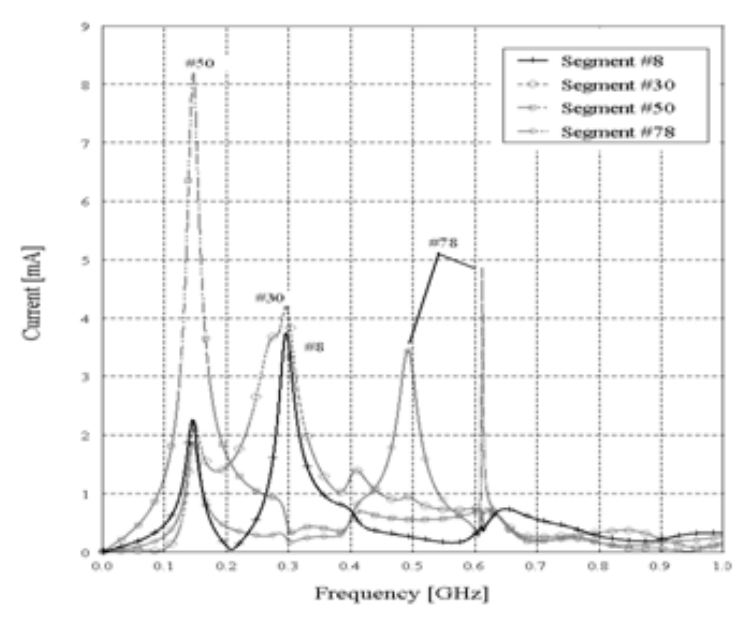

Figure 5: Current-frequency responses of the selected segments belonging to the nose (\#8), wing(\#30), $\operatorname{mid}(\# 50)$, and tail stabilizer (\#78), respectively.

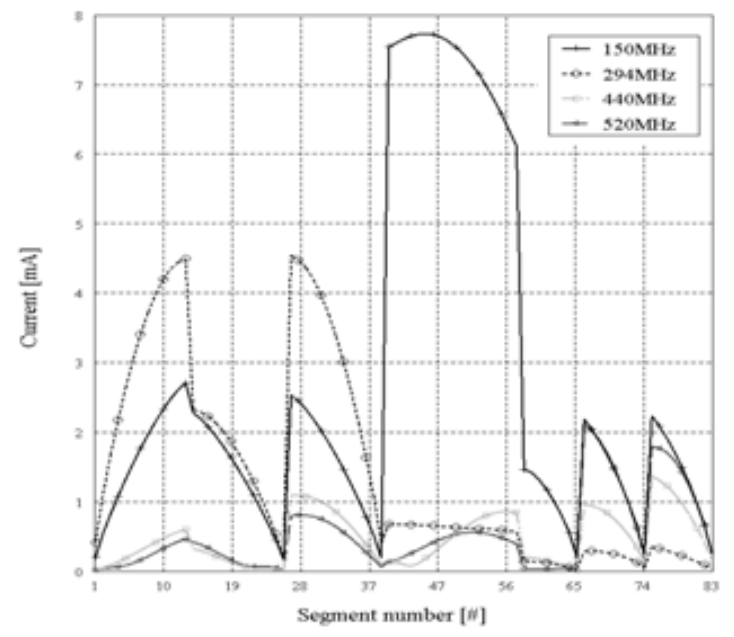

Figure 6: Current strength per segments for the set of discrete resonant frequencies
After applying the MPOF to the temporal backscattered signaldepicted inFigure 7, a series of four resonances exist at approximately the following frequencies: 150.4, 294.9, 441.5 and $519.5 \mathrm{MHz}$.Compared toFigure 5, the resonance peaks in the frequency response and the extracted ones closely coincide, which confirms their relation to themid, the nose-wings, the wings and the tails sections, respectively. Next, the first model complex residues results are shown in Table II, then inserting the complex residues into (5) for each and then inserting each respective $K_{c}$ into (8) and applying (10) and solving, the co-pol CPS set at each resonance is derived and listed inTable III.

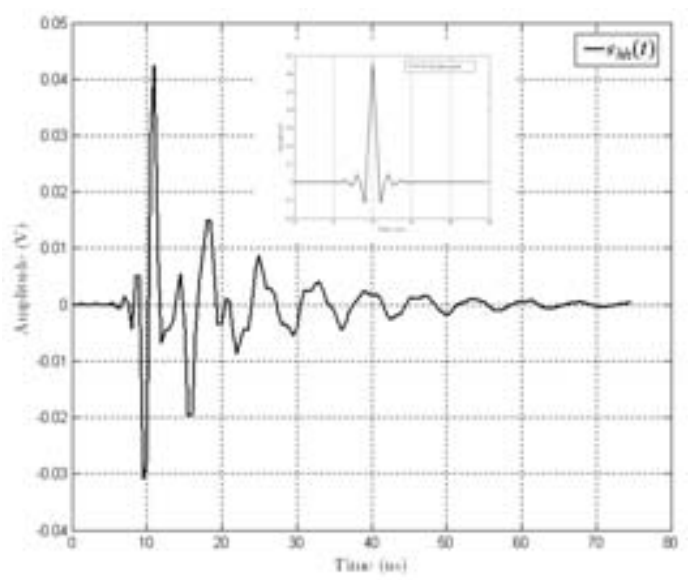

Figure 7:Backscattered transient response of the aircraft model. Inset: the incident waveform.

Table II:The Scattering coefficients in terms of the Complex residues and their NRFs

\begin{tabular}{|c|c|c|c|}
\hline Mode order & $c_{\mathrm{xx}}$ & $c_{\mathrm{yx}}$ & $c_{\mathrm{yv}}$ \\
\hline 1 & $0.49-.13 \mathrm{j}$ & $0.37-0.38 \mathrm{j}$ & $-0.49+0.13 \mathrm{j}$ \\
\hline 2 & $0.92+073 \mathrm{j}$ & $0.28+0.11 \mathrm{j}$ & $-0.92-0.73 \mathrm{j}$ \\
\hline 3 & $-1.44+3.64 \mathrm{j}$ & $0.64+0.03 \mathrm{j}$ & $1.44-3.64 \mathrm{j}$ \\
\hline 4 & $-0.97-2.23 \mathrm{j}$ & $-0.65+0.15 \mathrm{j}$ & $-0.97-2.23 \mathrm{j}$ \\
\hline
\end{tabular}

Table IIIindicates that the target has different CPS set at each resonance, and subsequently, different physical attributes. At the first resoanance, the geometry is forcasted to be highly long as the dot product $\mathrm{g}_{\mathrm{cn} 1} \cdot \mathrm{g}_{\mathrm{cn} 2}=1$ and tilted about $\quad 45^{\circ}$ (as $\quad 1 / 2 \tan ^{-1}\left(\mathrm{~g}_{2} / \mathrm{g}_{1}\right)=45^{\circ}$ for $\left.\left[\mathrm{g}_{\mathrm{cn} 1}+\mathrm{g}_{\mathrm{cn} 2}\right]\right)$; thus corresponding mainly to the mid section. As for the second to the fourth, they have similar CPS sets with non-linear property as $\mathrm{g}_{\mathrm{cn} 1} \mathrm{~g}_{\mathrm{cn} 2} \neq 1$, forecasting a dihedral structure. Importantly, the $\mathrm{g}_{3}$ of the co-max set $\mathrm{cm}_{1,2}$ for all resonances are zero, indicating that all target geometries are absolutely symterical. These attributes of the CPS at the dominant resonances totally agree with what is a priori known about the target composite. Next, the ability of the co-null state to discriminate between two similar aircrafts models is demonstrated. For a model with $90^{\circ}$ inclined wings and tails, the CPS set at each resonance is derived and listed inTable IV. Comparing the results of both tables for the $45^{\circ}$ and $90^{\circ}$ models; their co-null set at the second to forth resonance demonstrate the major difference. This complies with the change of inclination angle made to the wings and tails. 


\section{International Journal of Science and Research (IJSR) \\ ISSN (Online): 2319-7064}

Index Copernicus Value (2013): 6.14 | Impact Factor (2015): 6.391

\begin{tabular}{|c|c|c|c|c|c|c|}
\hline $\begin{array}{l}\text { Mode } \\
\text { order }\end{array}$ & CPS & $g_{1}$ & $g_{2}$ & $g_{3}$ & $P$ & $\begin{array}{c}\text { Forecasted } \\
\text { Shape }\end{array}$ \\
\hline \multirow{4}{*}{1} & $\mathrm{~cm}_{1}$ & 0 & 1 & 0 & 1 & \multirow{4}{*}{ long at +45} \\
\hline & $\mathrm{cm}_{2}$ & 0 & -1 & 0 & 0 & \\
\hline & $\mathrm{cn}_{1}$ & $\mathbf{0}$ & -1 & $\mathbf{0}$ & $\mathbf{0}$ & \\
\hline & $\mathrm{cn}_{2}$ & $\mathbf{0}$ & -1 & $\mathbf{0}$ & $\mathbf{0}$ & \\
\hline \multirow{4}{*}{2} & $\mathrm{~cm}_{1}$ & 0 & 1 & 0 & 1 & \multirow{4}{*}{ Dihedral at +45} \\
\hline & $\mathrm{cm}_{2}$ & 0 & -1 & 0 & 0.34 & \\
\hline & $\mathrm{cn}_{1}$ & $\mathbf{0}$ & -0.26 & 0.97 & $\mathbf{0}$ & \\
\hline & $\mathbf{c n}_{2}$ & 0 & -0.26 & -0.97 & 0 & \\
\hline \multirow{4}{*}{3} & $\mathrm{~cm}_{1}$ & 0 & 1 & 0 & 1.0 & \multirow{4}{*}{ Dihedral at +45} \\
\hline & $\mathrm{cm}_{2}$ & 0 & -1 & 0 & 0.51 & \\
\hline & $\mathrm{cn}_{1}$ & $\mathbf{0}$ & -0.16 & 0.98 & $\mathbf{0}$ & \\
\hline & $\mathrm{cn}_{2}$ & $\mathbf{0}$ & -0.16 & -0.98 & $\mathbf{0}$ & \\
\hline \multirow{4}{*}{4} & $\mathrm{~cm}_{1}$ & 0 & 1 & 0 & 1 & \multirow{4}{*}{ Dihedral at +45} \\
\hline & $\mathrm{cm}_{2}$ & 0 & -1.0 & 0.0 & 0.32 & \\
\hline & $\mathrm{cn}_{1}$ & $\mathbf{0}$ & -0.27 & 0.96 & 0.0 & \\
\hline & $\mathrm{cn}_{2}$ & $\mathbf{0}$ & -0.27 & -0.96 & 0.0 & \\
\hline
\end{tabular}

\section{References}

[1] H. S. Lui and N. V. Z. Shuley, "Radar Target Identification Using a "Banded" E-pulse Technique," Antennas and Propagation, IEEE Transactions on, vol. 54, pp. 3874-3881, 2006.

[2] D. Lan, L. Hongwei, B. Zheng, and Z. Junying, "A twodistribution compounded statistical model for Radar HRRP target recognition," IEEE Transactions on Signal Processing, , vol. 54, pp. 2226-2238, 2006.

[3] W. Dangwei, M. Xinyi, and S. Yi, "Radar target identification using a likelihood ratio test and matching pursuit technique," Radar, Sonar and Navigation, IEE Proceedings -, vol. 153, pp. 509-515, 2006.

[4] K.-T. Kim, D.-K. Seo, and H.-T. Kim, "Radar target identification using one-dimensional scattering centres," Radar, Sonar and Navigation, IEE Proceedings -, vol. 148, pp. 285-296, 2001.

Table IV:Compared to first model, The CPS results of the second Model depict co-null discriminative ability.

\begin{tabular}{|c|c|c|c|c|c|}
\hline Mode order & CPS & $g_{1}$ & $g_{2}$ & $g_{3}$ & $P$ \\
\hline \multirow{4}{*}{1} & $\mathrm{~cm}_{1}$ & 0.0 & 1.0 & 0.0 & 1.0 \\
\hline & $\mathrm{cm}_{2}$ & 0.0 & -1.0 & 0.0 & 0.0 \\
\hline & $\mathrm{cn}_{1}$ & $\overline{0.0}$ & -1.0 & 0.0 & 0.0 \\
\hline & $\mathrm{cn}_{2}$ & $\overline{0.0}$ & -1.0 & 0.0 & $\mathbf{0 . 0}$ \\
\hline \multirow{4}{*}{2} & $\mathrm{~cm}_{1}$ & 0.0 & 1.0 & 0.0 & 1.0 \\
\hline & $\mathrm{cm}_{2}$ & 0.0 & -1.0 & 0.0 & 0.03 \\
\hline & $\mathrm{cn}_{1}$ & $\overline{0.0}$ & -0.72 & 0.7 & 0.00 \\
\hline & $\mathrm{cn}_{2}$ & $\begin{array}{l}0.0 \\
\end{array}$ & $\begin{array}{c}-0.72 \\
\end{array}$ & -0.7 & 0.00 \\
\hline \multirow{4}{*}{3} & $\mathrm{~cm}_{1}$ & 0.0 & 1.0 & 0.0 & 1.0 \\
\hline & $\mathrm{cm}_{2}$ & 0.0 & -1.0 & 0.0 & 0.71 \\
\hline & $\mathrm{cn}_{1}$ & 0.0 & -0.08 & 1.0 & 0.0 \\
\hline & $\mathrm{cn}_{2}$ & $\overline{0.0}$ & -0.08 & -1.0 & 0.0 \\
\hline \multirow{4}{*}{4} & $\mathrm{~cm}_{1}$ & 0.0 & 1.0 & 0.0 & 1.0 \\
\hline & $\mathrm{cm}_{2}$ & 0.0 & -1.0 & 0.0 & 0.0 \\
\hline & $\mathrm{cn}_{1}$ & 0.0 & -0.87 & 0.49 & $\mathbf{0 . 0}$ \\
\hline & $\mathrm{cn}_{2}$ & 0.0 & -0.87 & -0.49 & 0.0 \\
\hline
\end{tabular}

\section{Conclusions}

Physical attributes about the target composition is well contained in the characteristics co-null states of its resonance modes. The co-null set reveals the degrees of elongation and tilt in the target composition. By this comparison of two slightly different shaped aircraft models, it is safe to conclude that the co-null established at resonance modes has robust discriminative ability, and consequently, can be utilized to derive a robust feature set for the purpose of target identification. Henceforth, the concept of co-null states is established in a resonance mode context. Finally, implementing an identification algorithm based on this feature set, and subsequently, assessing its performance with target aspect variation and in presence of noise can be a subject of further studies.

\section{Acknowledgment}

This work was supported and funded by The Public Authority of Education and Training, Research project No (TS-11-14).

[5] D. A. Garren, A. C. Odom, M. K. Osborn, J. S. Goldstein, S. U. Pillai, and J. R. Guerci, "Fullpolarization matched-illumination for target detection and identification," Aerospace and Electronic Systems, IEEE Transactions on, vol. 38, pp. 824-837, 2002.

[6] W. M. Steedly and R. L. Moses, "High resolution exponential modeling of fully polarized radar returns," Aerospace and Electronic Systems, IEEE Transactions on, vol. 27, pp. 459-469, 1991.

[7] P. B. Silverstein, O. S. Sands, and F. D. Garber, "Radar target classification and interpretation by means of structural descriptions of backscatter signals," Aerospace and Electronic Systems Magazine, IEEE, vol. 6, pp. 3-7, 1991.

[8] F. Aldhubaib, N. V. Shuley, and H. S. Lui, "Characteristic Polarization States in an Ultrawideband Context Based on the Singularity Expansion Method," IEEE Geoscience and Remote Sensing Letters, vol. 6, pp. 792-796, 2009.

[9] FA Sadjadi, CSL Chun, A Sullivan, and G. Gaunaurd, "The Huynen-Fork Polarization Parameters in the Classification of Dielectric Mine-like Objects," Sensing and Imaging: An International Journal, vol. 7, 2006.

[10] W.-M. Boerner, W.-L. Yan, A.-Q. Xi, and Y. Yamaguchi, "On the basic principles of radar polarimetry: the target characteristic polarization state theory of Kennaugh, Huynen's polarization fork concept, and its extension to the partially polarized case," Proceedings of the IEEE, vol. 79, pp. 1538-1550, 1991.

[11] J. Yang, Y. Yamaguchi, H. Yamada, Z. H. Czyz, W.-M. Boerner, H. Mott, et al., "The characteristic polarization states and the equi-power curves," Geoscience and Remote Sensing, IEEE Transactions on, vol. 40, pp. 305-313, 2002.

[12]Y. Yamaguchi, W.-M. Boerner, H. J. Eom, M. Sengoku, S. Motooka, and T. Abe, "On characteristic polarization states in the cross-polarized radar channel," Geoscience and Remote Sensing, IEEE Transactions on, vol. 30, pp. 1078-1080, 1992.

[13]W. M. Boerner, W. L. Yan, A. Q. Xi, and Y. Yamaguchi, "The characteristic polarization states for the coherent and partially polarized case," in Antennas and Propagation, 1991. ICAP 91., Seventh 
International Conference on (IEE), 1991, pp. 383-391 vol.1.

[14]H. Mott, Remote sensing with polarimetric radar New York, N.Y: Wiley-IEEE ; Chichester : John Wiley 2007.

[15] Fawwaz T. Ulaby and C. Elachi, Radar polarimetry for geoscience applications: Norwood, Mass. : Artech House, 1990.

[16]C. E. Baum, E. J. Rothwell, K.-M. Chen, and D. P. Nyquist, "The singularity expansion method and its application to target identification," Proceedings of the IEEE, vol. 79, pp. 1481-1492, 1991.

[17]C. E. Baum, "Signature-based target identification and pattern recognition," Antennas and Propagation Magazine, IEEE, vol. 36, pp. 44-51, 1994.

[18]F. F. H. Aldhubaib and N. V. Z. Shuley, "Characteristic Polarization States Estimation in an Ultrawideband Context: A Frequency Approach," IEEE Transactions on Geoscience and Remote Sensing, vol. 47, pp. 28082817, 2009.

[19]F. Aldhubaib, N. V. Shuley, and I. D. Longstaff, "On the application of pattern recognition to identification of simple targets based on resonance and polarization diversity," in Radar Systems, 2007 IET International Conference on, 2007, pp. 1-5.

[20]F. Aldhubaib and N. V. Shuley, "Radar Target Recognition Based on Modified Characteristic Polarization States," IEEE Transactions on Aerospace and Electronic Systems, vol. 46, pp. 1921-1933, 2010.

[21]F. Aldhubaib, H. S. Lui, N. V. Shuley, and A. AlZayed, "Aspect segmentation and feature selection of radar targets based on average probability of error," IET Microwaves, Antennas \& Propagation, vol. 4, pp. 16541664, 2010.

[22]F. Aldhubaib, "Polarization Angles As A Radar Feature Set " International Journal of Enhanced Research in Science Technology \& Engineering (IJERSTE), vol. 5, April - 20162016.

[23]T. K. Sarkar and O. Pereira, "Using the matrix pencil method to estimate the parameters of a sum of complex exponentials," Antennas and Propagation Magazine, IEEE, vol. 37, pp. 48-55, 1995.

[24]E. s. a. systems, "Feko Suit 5," 9.3.24 ed. S.A (Pty) Ltd, 2003-2005.

\section{Author Profile}



Faisal F. H. Aldhubaib received his B.E. degree in electrical and computer engineering and M.Scdegreein radio and microwave engineering from the University Of Leeds, UK, in 1995 and 1997, respectively. Then in 2010, he received his $\mathrm{PhD}$ in Electromagnetic and Imaging Research Group at the School of Information Technology and Electrical Engineering (ITEE) at the University of Queensland, Australia. Currently he is an assistant professor working in the college of technological studies, PAAET, Kuwait. 